\title{
SLEEP APNEA AS A RISK FACTOR FOR DIASTOLIC DYSFUNCTION: A SYSTEMATIC REVIEW AND META-ANALYSIS
}

Mohammed Al-Sadawi ${ }^{1}$, Kleanthis Theodoropoulos ${ }^{2}$, farzane saeidifard ${ }^{2}$, Adekunle Kiladejo $^{2}$, Patricia Erwin ${ }^{2}$, Mohammad Al-Ajam², Louis Salciccioli ${ }^{3}$, and Adam Budzikowski ${ }^{2}$

${ }^{1}$ Stony Brook Medicine

${ }^{2}$ Affiliation not available

${ }^{3}$ SUNY Downstate Medical Center

April 18, 2021

\begin{abstract}
Abstract: Background: This meta-analysis assessed the relationship between Obstructive Sleep Apnea (OSA) and echocardiographic parameters of diastolic dysfunction (DD), which are used in the assessment of Heart Failure with Preserved Ejection Fraction (HFpEF). Methods: We searched the databases including Ovid MEDLINE, Ovid Embase Scopus, Web of Science, Google Scholar, and EBSCO CINAHL from inception up to December 26th, 2020. The search was not restricted to time, publication status or language. Comparisons were made between patients with OSA, diagnosed in-laboratory polysomnography (PSG) or home sleep apnea testing (HSAT), and patients without OSA in relation to established markers of diastolic dysfunction. Results: Primary search identified 2512 studies. A total of 18 studies including 2509 participants were included. The two groups were free of conventional cardiovascular risk factors. Significant structural changes were observed between the two groups. Patients with OSA exhibited greater LAVI (3.94 CI [0.8, 7.07]; $\mathrm{p}=0.000)$ and left ventricular mass index (11.10 CI $[2.56,19.65] ; \mathrm{p}=0.000)$ as compared to control group. The presence of OSA was also associated with more prolonged DT (10.44 ms CI [0.71,20.16]; $\mathrm{p}=0.04)$, IVRT (7.85 ms CI[4.48, 11.22]; $\mathrm{p}=0.000)$, and lower E/A ratio $(-0.62 \mathrm{CI}[-1,-0.24] ; \mathrm{p}=0.001)$ suggestive of early DD. The E/e' ratio $(0.94 \mathrm{CI}[0.44,1.45] ; \mathrm{p}=0.000)$ was increased. Conclusion: An association between OSA and echocardiographic parameters of DD was detected that was independent of conventional cardiovascular risk factors. OSA may be independently associated with DD perhaps due to higher LV mass. Investigating the role of CPAP therapy in reversing or ameliorating diastolic dysfunction is recommended.
\end{abstract}

\section{SLEEP APNEA AS A RISK FACTOR FOR DIASTOLIC DYSFUNCTION: A SYSTEMATIC REVIEW AND META-ANALYSIS}

Keywords: Sleep Apnea, diastolic dysfunction, systematic review, meta-analysis.

Mohammed Al-Sadawi, M.D., ${ }^{1}$ Kleanthis Theodoropoulos, M.D., ${ }^{2}$ Farzane Saeidifard, M.D., ${ }^{3,4}$ Adekunle Kiladejo, M.D., ${ }^{2}$ Patricia Erwin, ${ }^{4}$ Mohammad Al-Ajam, M.D., ${ }^{5}$ Louis Salciccioli, M.D. ${ }^{2}$ Adam S Budzikowski, M.D., PhD, FHRS ${ }^{2}$

${ }^{1}$ Department of Cardiovascular Medicine, Stony Brook medicine, Stony brook, New York, United States

${ }^{2}$ Division of Cardiovascular Medicine; Department of Medicine. SUNY Downstate, Brooklyn, New York, United States 
${ }^{3}$ Department of Internal Medicine, Lenox Hill Hospital, Northwell Health, Zucker School of Medicine, New York, United States

${ }^{4}$ Division of Preventive Cardiology, Department of Cardiovascular Medicine, Mayo Clinic, Rochester, Minnesota, United States

${ }^{5}$ Division of Pulmonary and Critical Care Medicine, Department of Veterans Administration NY Harbor Healthcare System, Brooklyn Campus, Brooklyn, New York, United States

\section{Correspondence:}

Mohammed Al-Sadawi, MD

Department of Cardiovascular Medicine

Stony Brook medicine,

Stony brook, New York, United States

Mohammed.al-sadawi@stonybrookmedicine.edu

Abstract:

Background: This meta-analysis assessed the relationship between Obstructive Sleep Apnea (OSA) and echocardiographic parameters of diastolic dysfunction (DD), which are used in the assessment of Heart Failure with Preserved Ejection Fraction (HFpEF).

Methods: We searched the databases including Ovid MEDLINE, Ovid Embase Scopus, Web of Science, Google Scholar, and EBSCO CINAHL from inception up to December 26th, 2020. The search was not restricted to time, publication status or language. Two independent investigators screened the identified studies and extracted the data, in duplicate. We conducted a meta-analysis using RevMan v.5. Risk of bias was assessed using Cochrane collaboration tools. Comparisons were made between patients with OSA, diagnosed in-laboratory polysomnography (PSG) or home sleep apnea testing (HSAT), and patients without OSA in relation to established markers of diastolic dysfunction.

\section{Results:}

Primary search identified 2512 studies. A total of 18 studies including 2509 participants were included. The two groups were free of conventional cardiovascular risk factors. Significant structural changes were observed between the two groups. Patients with OSA exhibited greater LAVI (3.94 CI [0.8, 7.07]; $\mathrm{p}=0.000)$ and left ventricular mass index (11.10 CI $[2.56,19.65] ; \mathrm{p}=0.000)$ as compared to control group. The presence of OSA was also associated with more prolonged DT (10.44 ms CI [0.71,20.16]; p=0.04), IVRT (7.85 ms $\mathrm{CI}[4.48,11.22] ; \mathrm{p}=0.000)$, and lower $\mathrm{E} / \mathrm{A}$ ratio $(-0.62$ CI $[-1,-0.24] ; \mathrm{p}=0.001)$ suggestive of early DD. The $\mathrm{E} / \mathrm{e}$ ' ratio $(0.94 \mathrm{CI}[0.44,1.45] ; \mathrm{p}=0.000)$ was increased. Linear correlation between severity of OSA and LAVI and IVRT parameters was observed but this association did not sustain for the E/A and E/e'. The ejection fraction was not significantly different between patients with OSA and healthy controls (-0.48 CI $[-1.18,0.23] ; \mathrm{p}=0.18)$.

Conclusion: An association between OSA and echocardiographic parameters of DD was detected that was independent of conventional cardiovascular risk factors. OSA may be independently associated with DD perhaps due to higher LV mass. Investigating the role of CPAP therapy in reversing or ameliorating diastolic dysfunction is recommended.

Introduction:

Diastolic heart failure, or heart failure with preserved ejection fraction (HFpEF) accounts for approximately $50 \%$ of all heart failure and it is associated with increased morbidity and mortality similar to systolic heart failure (1). It has been suggested that DD is the result of a response to systemic inflammation predisposed by multiple comorbidities such as obesity, hypertension, and diabetes that may lead to remodeling of the 
myocardium, signaling dysfunction and finally cardiomyocyte hypertrophy, myocardial stiffness and progressive interstitial fibrosis (2). Noteworthy, DD has been found to be an independent predictor of mortality in patients with normal left ventricular ejection fraction (1). Therefore, the early detection of susceptible persons and the identification of new risk factors for DD and HFpEF and attempting to control such conditions is crucial for the management of these patients.

Although observational data suggest a bidirectional relationship between obstructive sleep apnea (OSA) and HFpEF (3), it remains unknown whether this association is due to OSA itself or is mediated by the higher burden of co-existing cardio-metabolic disorders frequently seen in OSA patients.

OSA is a chronic respiratory sleep disorder characterized by repetitive apnea/hypopnea episodes induced by narrowing of the upper airway during sleep. The intermittent episodes of hypoxemia and sympathetic overdrive shown to be associated with these apneas and hypopneas lead to endothelial dysfunction, systemic inflammation, oxidative stress, arterial hypertension exerting deleterious effects in the cardiovascular system. OSA has been associated with increased risk for coronary artery disease, heart failure, arrhythmias, and pulmonary hypertension (4).

Although previous studies have suggested an association between OSA and DD, the majority of them were limited by the retrospective nature, small sample size, and the high prevalence of clinical confounders including hypertension, obesity, and diabetes that these patients exhibit. Therefore, in this meta-analysis we sought to investigate the correlation between DD and OSA in patients free of conventional cardiovascular risk factors.

Methods:

\section{Literature search and data sources}

This systematic review and meta-analysis were executed using a preplanned protocol. The results were reported according to the Preferred Reporting Items for Systematic Reviews and Meta-Analyses (PRISMA) guidelines(5). An electronic search with the assistance of a librarian was conducted. We searched the databases including Ovid MEDLINE, Ovid Embase Scopus, Web of Science, Google Scholar, and EBSCO CINAHL from inception up to December 26th, 2020 were queried. The search was not restricted to time, publication status or language. Moreover, the references as well as studies related to first author and corresponding author of included studies were hand-searched for other relevant studies. We used Medical Subject Headings (MeSH) terms with keywords sleep apnea, sleep-disordered breathing, hypoventilation disorder/disease, DD and heart failure with preserved/normal ejection fraction. (Figure 1) shows details. We included randomized and quasi-randomized designs, pre-post trials, cross-sectional studies, and observational studies.

\section{Study selection and quality assessment}

Two reviewers (MA and AK) screened independently and blindly the potential eligibility of each of the abstracts and titles that result from executing the search strategy using a systematic review software (Covidence, London, UK). The reviewers evaluated the abstracts for inclusion and exclusion criteria, in case that all are not disclosed in abstract methods, the study was classified as potentially eligible and the complete article was examined to determine inclusion. Subsequently, disagreements were solved by consensus. Then, both reviewers evaluated the full text versions of all potentially eligible studies. Inclusion criteria are: (1) studies contains data of echocardiographic parameters in two groups; group with sleep apnea disorder and a control group (2) studies with data of echocardiographic parameters in different groups with varying sleep apnea severity.

Echocardiographic parameters needed for inclusion were prespecified according to the update published in 2016 by the American Society of Echocardiography (ASE) and the European Association of Cardiovascular Imaging (EACVI) for the evaluation of Left Ventricular Diastolic Function by Echocardiography(6). Any study with at least one parameter was included. The parameters commonly found were mitral valve deceleration time, isovolumic relaxation time, the ratio of early mitral inflow velocity to mitral annular early diastolic 
velocity E/e', left atrial volume index (>34 mL/m2), the ratio of early to late mitral inflow velocities E/A $(>14)$, and left ventricular mass index.

Diagnosis of sleep apnea was considered according to the guidelines of American Academy of Sleep Medicine Clinical Practice Guideline (7). The sleep disorder group consisted of symptomatic patients who had an inlaboratory polysomnography (PSG) or home sleep apnea testing (HSAT) confirmation of sleep apnea with an apnea hypopnea index (AHI) greater than 5 .

\section{Data extraction}

Data extraction was done by two reviewers (MA, FS) with discrepancies resolved by consensus. Data included: first author, date of publishing, eligibility criteria, methods, risk of bias assessment, participants, intervention and control groups, outcomes, and results (dichotomous or continuous outcomes). Original authors were contacted, if needed, for non-published or incomplete data.

\section{Statistical analysis}

The meta-analysis was performed using Review Manager, version 5 (Cochrane Collaboration). Mean differences were evaluated along with $95 \%$ confidence intervals (95\% CIs). All parameters were summarized using the random-effects model. Heterogeneity, Cochran's Q, tau-squared test, and I2 index were assessed for each study. Risk of bias was assessed using Cochrane collaboration tools.

Results:

Our primary search identified potentially eligible 2512 studies. After the removal of duplicates and screening of titles and abstracts, 54 studies underwent full -text review. After screening references and authors, a total of 18 studies met all eligibility criteria and were included in our meta-analysis. 2509 participants were included. (Figure 2)(7-23)

\section{Patient Characteristics}

The mean age and Body Mass Index (BMI) showed no statistical difference between the obstructive sleep apnea patients (58.8 \pm 11 years; $29.12 \pm 5.11$, respectively) and the control group (55.06 \pm 11 years, $26.7 \pm 4.4$ respectively), ( $>0.05)$. Demographic data, blood pressure, diabetes and lipid profile of the participants are summarized in Table 1.

\section{Relation with DD}

For DD echocardiographic parameters, OSA group had higher values in deceleration time than the control group (10.44 ms CI $[0.71,20.16] ; \mathrm{p}=0.04)$. Isovolumic relaxation time was statistically prolonged in OSA group $(7.85 \mathrm{~ms}$ CI $[4.48,11.22] ; \mathrm{p}=0.000)$. The ratio of early mitral inflow velocity to mitral annular early diastolic velocity $\mathrm{E} / \mathrm{e}$ ' $(0.94 \mathrm{CI}[0.44,1.45] ; \mathrm{p}=0.000)$ and left atrial volume index $(3.94 \mathrm{CI}[0.8,7.07] ; \mathrm{p}=0.000)$ were significantly higher in OSA group. The ratio of early to late mitral inflow velocities (E/A ratio) was significantly lower in the OSA group compared to the control group $(-0.62$ CI $[-1,-0.24] ; \mathrm{p}=0.001)$. The LV mass index was also higher in OSA group compared to control group $(11.10 \mathrm{CI}[2.56,19.65] ; \mathrm{p}=0.000)$. Additionally, the systolic pulmonary artery pressure $(\mathrm{mmHg})$ was significantly higher in OSA group (8.06 CI $[0.94,15.17] ; \mathrm{p}=0.03)$. The left ventricular ejection fraction was not significantly different between patients with OSA and controls (-0.48 CI [-1.18,0.23]; p=0.18). Forest plots are shown in Figure 3.

\section{Relation between OSA severity and the degree of diastolic dysfunction}

Isovolumic relaxation time was significantly longer in severe vs mild OSA (mean difference $9.69 \mathrm{~ms}$ CI [5.16, 14.23]). The E/e' ratio was not significantly different between mild vs control but was higher in moderate to severe OSA vs control $(1.62[0.06,3.18])$. Left ventricular mass was significantly higher in moderate to severe vs mild OSA $(16.25 \mathrm{~g}[10.26,22.23])$. Although not statistically significant there was a trend towards inverse relationship between E/A ratio and severity of OSA, such that patients with more severe degree of sleep apnea had lower E/A ratio. Patients with moderate to severe sleep apnea tended to have greater LAVI as compared to controls or those with mild OSA but this association was not statistically significant. There was 
a trend toward higher left atrial volume index in both mild OSA vs control $(1.71[0.16,3.26])$ and moderate to severe vs control $(5.49[2.33,8.64])$ but the differences were not statistically significant. Forest plots are shown in Figure 4.

Discussion

To our knowledge this is the largest to date meta-analysis investigating the relationship between OSA and echocardiographic parameters of DD. An association between obstructive sleep apnea DD parameters was demonstrated which was independent of other risk factors.

Although cumulative data from observational studies have linked OSA with increased risk of DD, it has been argued that this association is driven mainly by the significant burden of cardiovascular risk factors observed in patients with OSA. To overcome this argument, we only included studies in which OSA patients were free from major cardiac risk factors. Therefore, our study results suggest a direct relationship between OSA and DD.

Of the numerous echocardiographic parameters mentioned, the American society of echocardiography (ASE) guidelines gives prominence to that the mitral inflow velocity ratios of E/A, e' as an estimation of LV filling pressure, left atrium volume index (LAVI), tricuspid regurgitation velocity and deceleration time in the assessment of the presence of DD. Two principal mechanisms are responsible for DD including impaired ventricular relaxation and increased passive myocardial stiffness (or decreased compliance). In grade I DD, there is impaired relaxation due to increased stiffness of the left ventricular chamber, resulting in slow early diastolic filling. LV relaxation is affected by load, inactivation, and asynchrony. Increased LV afterload leads to delayed and slow relaxation (6).

Sleep related breathing disorders including OSA exert a wide range of deleterious cardiovascular effects via direct and indirectly mechanisms. The repetitive episodes of apnea or hypopnea are associated with intermittent hypoxia, hypercapnia, increasingly negative intrathoracic pressure and sympathetic overdrive which all adversely affect the cardiovascular system. The hemodynamic and metabolic sequela of OSA result in endothelial dysfunction, increased inflammation, atherogenesis, hypertension, arrhythmias, and increased left ventricular transmural pressures. The combination of increased afterload and elevated heart rate contribute to left ventricular hypertrophy. Patients with OSA have been found to have increased levels of catecholamines and aldosterone suggesting activation of the renin-angiotensin-aldosterone axis (RAAS system), through the sympathetic overdrive, which in turn leads to cardiac fibrosis and remodeling contributing to impairment of myocardial relaxation, left ventricular stiffness and consequently to $\mathrm{DD}(3)$.

In agreement with the results of our analysis is the study by Butt et al (12) who provided a comprehensive assessment of LV structural and functional parameters using 2D and 3D echocardiography in moderatesevere OSA patients who were free of other concomitant confounder morbidities. The study included 120 otherwise healthy subjects with no evidence of cardiovascular disease, dyslipidemia or diabetes mellitus and were stratified into 3 groups based on the severity of sleep apnea and the presence of hypertension. The OSA group which included patients with AHI $>15$ and no hypertension $(n=40)$ were compared with matched disease control subjects who had essential hypertension but no OSA $(\mathrm{N}=40)$ and healthy control subjects who had neither hypertension nor OSA $(\mathrm{N}=40)$. It was demonstrated that patients with OSA and hypertension compared to the healthy subjects had higher left ventricular mass index and impaired systolic (decreased $\mathrm{S}$ systolic velocity) and diastolic function (E/A, IVRT, and E/e'). OSA patients were also found to have greater LAVI and lower ejection fraction as assessed by 3D echocardiography. Therapy with CPAP (mean duration of 6 months) resulted in significant structural changes (reduction in the LV mass and PWT/IVST) and improvement on the echocardiographic parameters of systolic and diastolic function.

On the contrary Kim et al (18) in a similar study with 62 patients classified into 3 groups (mild to moderate OSA, severe OSA, and control subjects) failed to detect significant correlation between OSA and DD. It was found that patients with OSA had decreased early diastolic velocity compared to control subjects but otherwise no differences in terms of $\mathrm{E} / \mathrm{A}$, isovolumic relaxation time, deceleration time, and pulmonary vein systolic/diastolic pulmonary vein velocity ratio were detected among the groups. Masa JF in a prospective 
study enrolled 196 patients with obesity hypoventilation syndrome and concomitant OSA treated with either NIV or CPAP. It was demonstrated that NIV and CPAP therapies improved left ventricular DD, reduced left atrial diameter, and pulmonary artery pressures at 3 years suggesting an association between sleep apnea and DD.

The prevalence of OSA has increased substantially in the past decade and it is expected to continue to rise due to the obesity epidemic, yet it is believed that OSA remains underdiagnosed (3). A major reason for underdiagnosis is that most patients with heart failure (HF) and sleep disordered breathing (SDB) do not complain of daytime sleepiness despite having significantly less sleep time. Another important factor that is that HF by itself or standard medications like beta-blockers in the treatment of HF can also cause fatigue masking SDB symptoms and thus offering an alternative to SDB leading to its underdiagnosis. This highlights the need to adopt a low threshold to test for sleep apnea in the context of HF.

With the increasing number of patients with HFpEF and the continued focus on reducing the rate of associated hospital admissions, comprehensive assessment of diastolic parameters and evaluation for those patients with HFpEF is an important aspect of cardiac testing. While all those with DD may not have HFpEF, when present this portends a worse prognosis. Grading of diastolic function is an important predictor of outcome. Longitudinal studies have shown an increased rate of all-cause mortality in patients with DD (24). Redfield and associates demonstrated an increased mortality risk in patients with DD when controlled for age, sex, and EF (25).

Therefore, it appears the early recognition of DD and HFpEF would be of clinical value, leading to more aggressive prevention and therapeutic strategies to ameliorate HF related symptoms or reduce disease progression. The association between OSA and DD underscores the need for increased level of awareness of such association among clinicians, and to have a low threshold for echocardiographic assessment of diastolic function.

To date the treatment of DD is treatment of the underlying etiology. The potential cardioprotective effects of CPAP or other therapy for OSA may offer a therapeutic tool for the treatment of DD in patients with OSA.

\section{Limitations}

Several limitations should be addressed when interpreting our results. First, our analysis consists of observational, non-randomized studies, which have inherent flaws in terms of selection bias and unmeasured confounders. Second, the current meta-analysis is based on study-level data; lack of patient level data precludes further subgroup analysis. Third, the lack of clinical outcomes limits conclusions regarding the long-term implications of this association. Fourth, there was significant heterogeneity among studies for many end point definitions such as OSA definition, severity, echocardiographic definition of DD. Fifth, the number of studies examining the correlation between DD and severity of sleep apnea were limited therefore definitive conclusions with regards to the association between DD and OSA severity would be precarious. In spite these limitations, the results of the present study can be used to inform future studies.

Conclusion

In our meta-analysis an association between OSA and DD was detected that appears independent of conventional cardiovascular risk factors. Investigation of OSA in HFpEF patients and conversely echocardiographic testing in OSA patients to screen for HFpEF is recommended. On the other hand, early application of OSA treatments might lead to more favorable outcomes in this therapeutically challenging clinical setting. Large prospective studies are warranted to demonstrate whether this approach confers more optimal long-term clinical outcomes. 
Search terms:

SEARCH STRATEGY: obstructive sleep apnea, obstructive sleep apnea syndrome, OSAHS, sleep apnea, sleep-disordered breathing, OSA, hypoventilation disorder, hypoventilation disease, obesity hypoventilation disorder, Sleep Apnea Hypopnea Syndrome, obesity hypoventilation disease, Sleep Apnea Hypopnea disorder, Sleep Apnea Hypopnea disease, Upper Airway Resistance Sleep Apnea Syndrome, Heart failure, $\mathrm{HF}$, heart failure with preserved ejection fraction, heart failure with normal ejection fraction, $\mathrm{HFpEF}$, diastolic heart failure, heart failure with diastolic disease, heart failure with diastolic disorder, heart failure with diastolic dysfunction.

[ obstructive sleep apnea OR obstructive sleep apnea syndrome OR OSAHS OR sleep apnea OR sleepdisordered breathing OR OSA OR, hypoventilation disorder OR hypoventilation disease OR, obesity hypoventilation disorder OR Sleep Apnea Hypopnea Syndrome OR obesity hypoventilation disease OR Sleep Apnea Hypopnea disorder R Sleep Apnea Hypopnea disease OR Upper Airway Resistance Sleep Apnea Syndrome]

AND

[Heart failure OR HF OR heart failure with preserved ejection fraction OR heart failure with normal ejection fraction OR HFpEF OR diastolic heart failure OR heart failure with diastolic disease OR heart failure with diastolic disorder OR heart failure with diastolic dysfunction]

Figure 1: Medical Subject Headings (MeSH) terms with keywords used for systematic review.

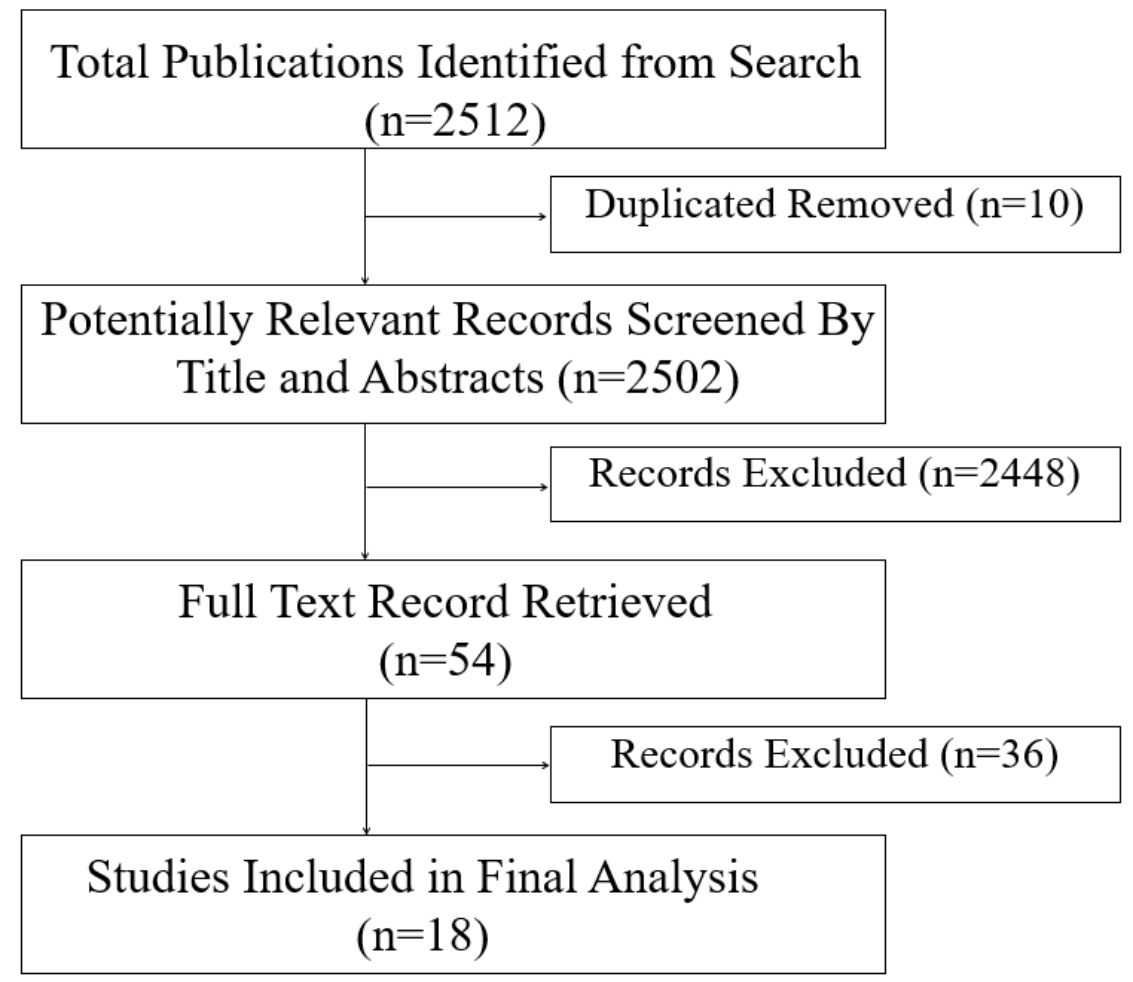

Figure 2: PRISMA flowchart describing the study's systematic literature search and study selection.

(in Excel sheet) 
Table1: Studies Characteristics and Demographic data of the participants.

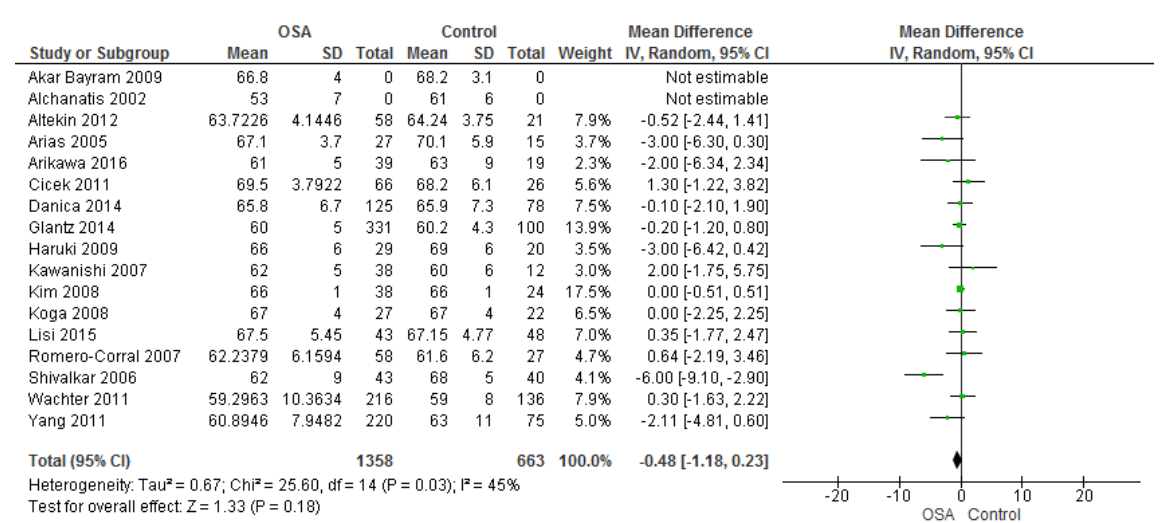

Forest plot of Odd ratios of left ventricular ejection fraction (LVEF) OSA vs non OSA.

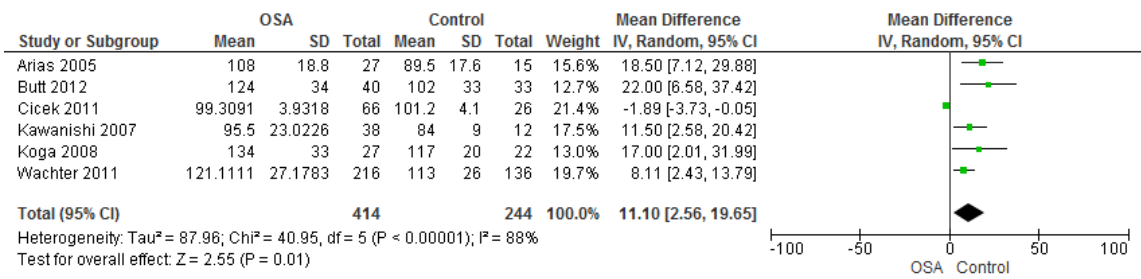

Forest plot of comparison: $1 \mathrm{HFpEF}$ in OSA vs non OSA, outcome: 1.10 LVMI (left ventricular mass index)

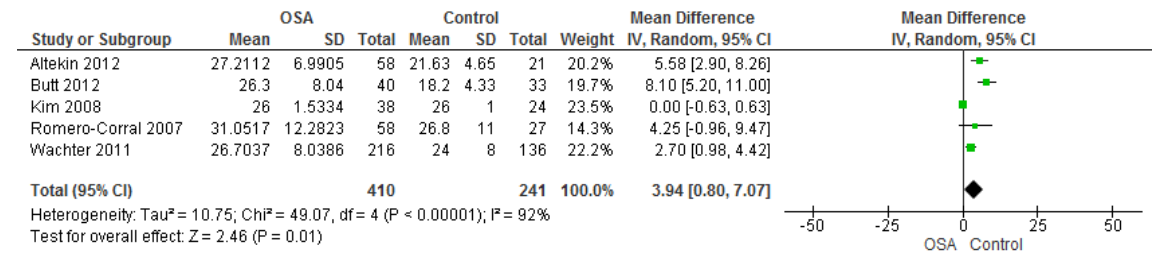

Forest plot of comparison: $1 \mathrm{HFpEF}$ in OSA vs non OSA, outcome: 1.13 LAVI (left atrial volume in$\operatorname{dex})(\mathrm{ml} / \mathrm{m} 2)$.

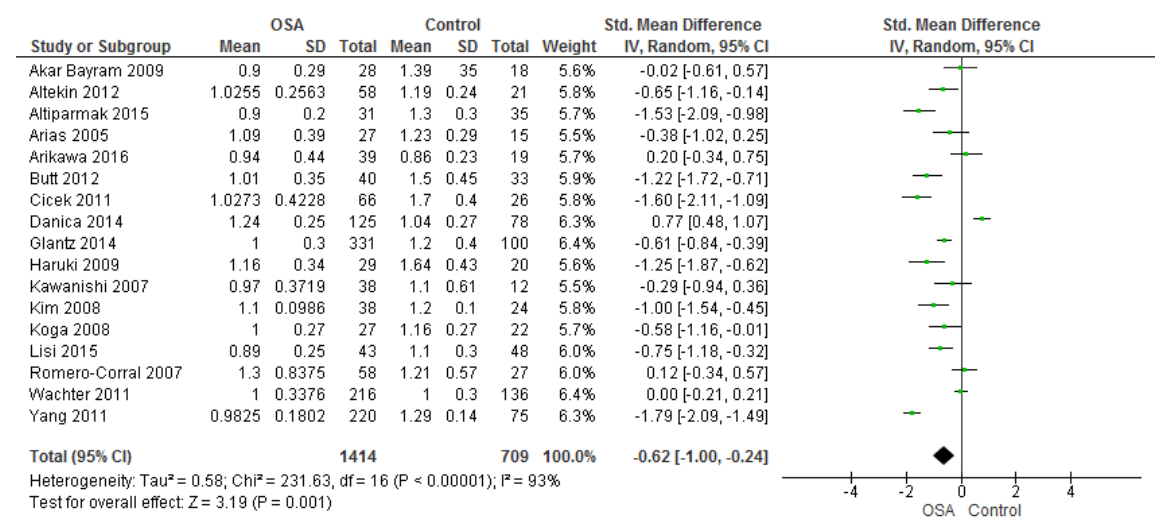


Forest plot of comparison: $1 \mathrm{HFpEF}$ in OSA vs non OSA, outcome: $1.14 \mathrm{E} / \mathrm{A}$ (ratio between diastolic early and late diastolic mitral inflow velocities).

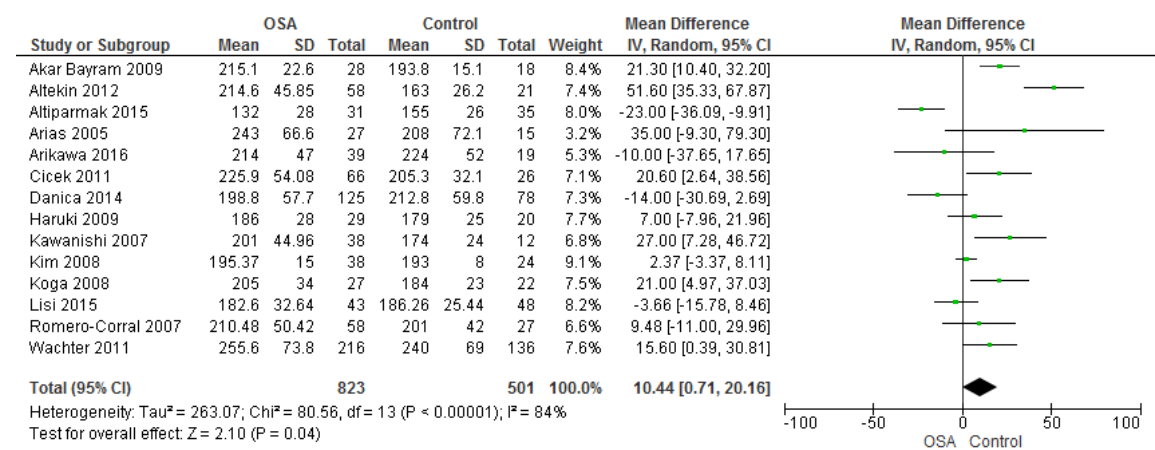

Forest plot of comparison: $1 \mathrm{HFpEF}$ in OSA vs non OSA, outcome: 1.15 DecT (deceleration time) (ms).

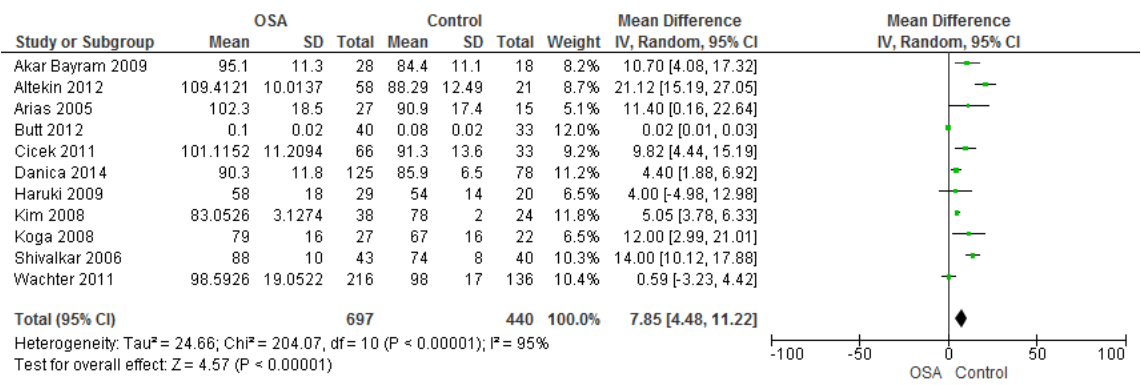

Forest plot of comparison: $1 \mathrm{HFpEF}$ in OSA vs non OSA, outcome: 1.16 IVRT (isovolumic relaxation time)(ms).

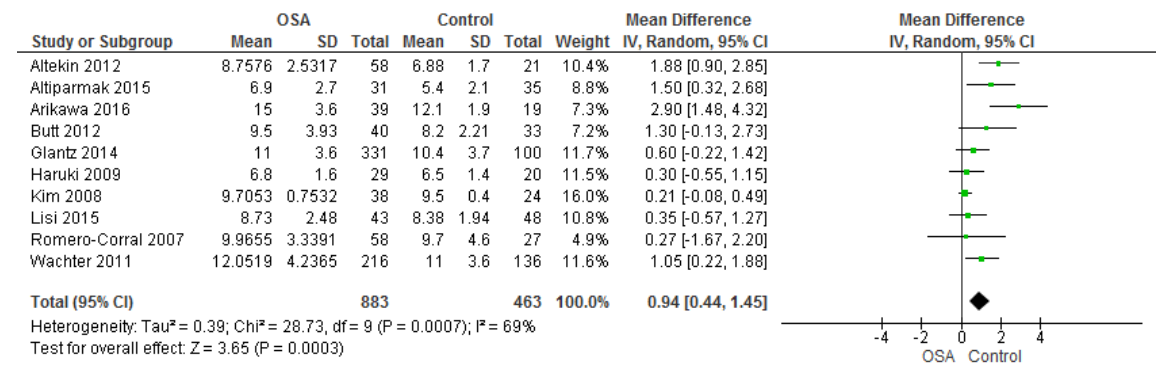

Forest plot of comparison: $1 \mathrm{HFpEF}$ in OSA vs non OSA, outcome: $1.17 \mathrm{E} / \mathrm{e}$ ' (ratio between early diastolic mitral inflow velocity and early diastolic annular velocity).

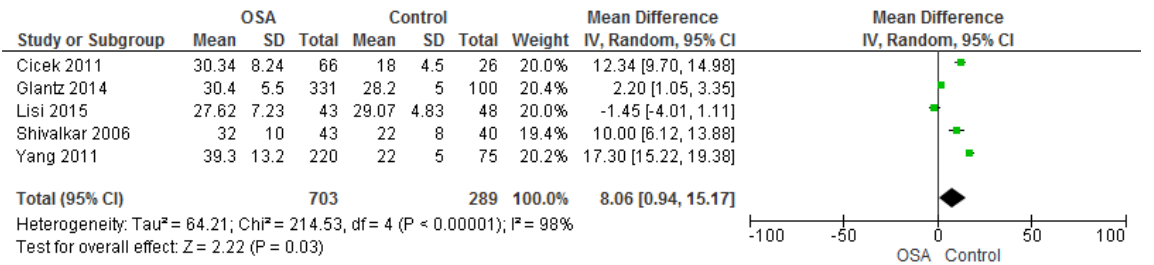


Forest plot of comparison: $1 \mathrm{HFpEF}$ in OSA vs non OSA, outcome: 1.18 SPAP ( systolic pulmonary artery pressure (mmHg).

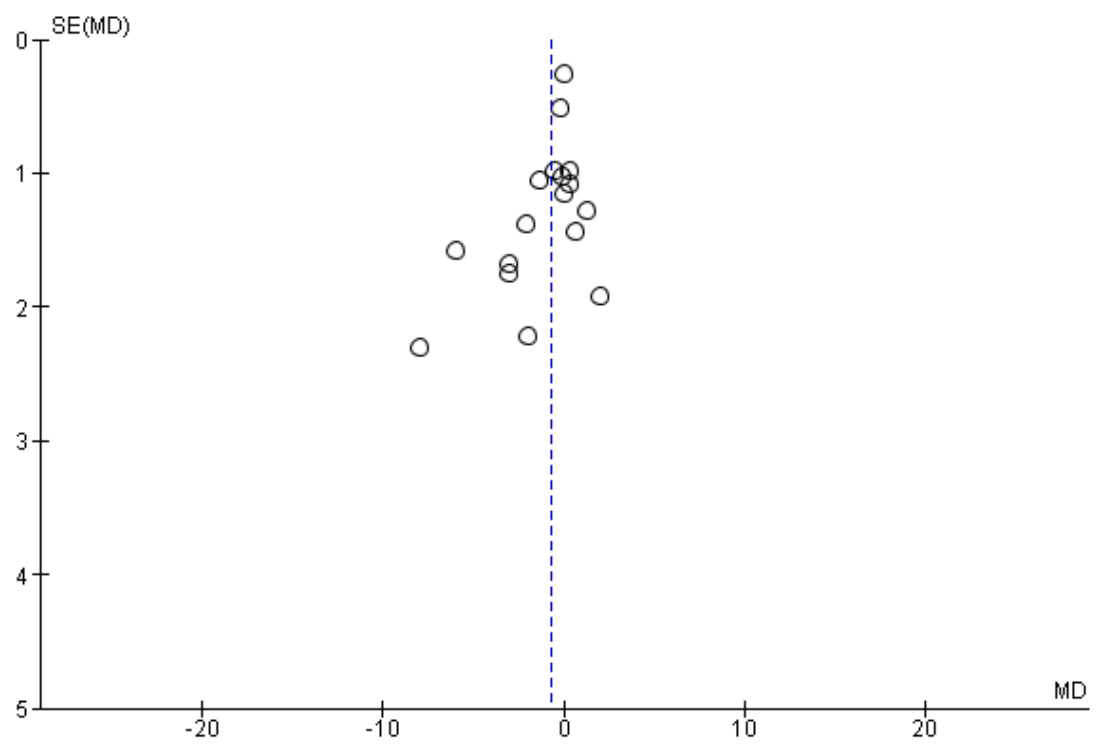

Funnel plot of comparison: 1 HFpEF in OSA vs non OSA, outcome: 1.1 LVEF.

Figure 3: Relationship between OSA and Echocardiographic parameters.

(In a separate word file)

Figure 4: Relationship between OSA severity and Echocardiographic parameters.

References:

1. Dunlay SM, Roger VL, Redfield MM. Epidemiology of heart failure with preserved ejection fraction. Nat Rev Cardiol. 2017;14(10):591-602.

2. Paulus WJ, Tschope C. A novel paradigm for heart failure with preserved ejection fraction: comorbidities drive myocardial dysfunction and remodeling through coronary microvascular endothelial inflammation. J Am Coll Cardiol. 2013;62(4):263-71.

3. Khattak HK, Hayat F, Pamboukian SV, Hahn HS, Schwartz BP, Stein PK. Obstructive Sleep Apnea in Heart Failure: Review of Prevalence, Treatment with Continuous Positive Airway Pressure, and Prognosis. Tex Heart Inst J. 2018;45(3):151-61.

4. Gonzaga C, Bertolami A, Bertolami M, Amodeo C, Calhoun D. Obstructive sleep apnea, hypertension and cardiovascular diseases. J Hum Hypertens. 2015;29(12):705-12.

5. Liberati A, Altman DG, Tetzlaff J, Mulrow C, Gotzsche PC, Ioannidis JP, et al. The PRISMA statement for reporting systematic reviews and meta-analyses of studies that evaluate healthcare interventions: explanation and elaboration. Bmj. 2009;339:b2700.

6. Nagueh SF, Smiseth OA, Appleton CP, Byrd BF, 3rd, Dokainish H, Edvardsen T, et al. Recommendations for the Evaluation of Left Ventricular Diastolic Function by Echocardiography: An Update from the American Society of Echocardiography and the European Association of Cardiovascular Imaging. Eur Heart J Cardiovasc Imaging. 2016;17(12):1321-60. 
7. Kapur VK, Auckley DH, Chowdhuri S, Kuhlmann DC, Mehra R, Ramar K, et al. Clinical Practice Guideline for Diagnostic Testing for Adult Obstructive Sleep Apnea: An American Academy of Sleep Medicine Clinical Practice Guideline. J Clin Sleep Med. 2017;13(3):479-504.

8. Altekin RE, Yanikoglu A, Baktir AO, Karakas MS, Ozel D, Cilli A, et al. Assessment of subclinical left ventricular dysfunction in obstructive sleep apnea patients with speckle tracking echocardiography. Int J Cardiovasc Imaging. 2012;28(8):1917-30.

9. Altiparmak IH, Erkus ME, Polat M, Yalcin F, Sak ZH, Sezen H, et al. Relation of elastic properties of pulmonary artery with left ventricular abnormalities and aortic stiffness in patients with moderate to severe obstructive sleep apnea: A cross-sectional echocardiographic study. Turk Kardiyol Dern Ars. 2016;44(4):28999.

10. Arias Miguel A, García-Río F, Alonso-Fernández A, Mediano O, Martínez I, Villamor J. Obstructive Sleep Apnea Syndrome Affects Left Ventricular Diastolic Function. Circulation. 2005;112(3):375-83.

11. Arikawa T, Toyoda S, Haruyama A, Amano H, Inami S, Otani N, et al. Impact of Obstructive Sleep Apnoea on Heart Failure with Preserved Ejection Fraction. Heart, Lung and Circulation. 2016;25(5):435-41.

12. Butt M, Dwivedi G, Shantsila A, Khair Omer A, Lip Gregory YH. Left Ventricular Systolic and Diastolic Function in Obstructive Sleep Apnea. Circulation: Heart Failure. 2012;5(2):226-33.

13. Cicek D, Lakadamyali H, Yagbasan BD, Sapmaz I, Muderrisoglu H. Obstructive sleep apnoea and its association with left ventricular function and aortic root parameters in newly diagnosed, untreated patients: a prospective study. J Int Med Res. 2011;39(6):2228-38.

14. Danica LP, Krotin M, Zdravkovic M, Soldatovic I, Zdravkovic D, Brajkovic M, et al. Early left ventricular systolic and diastolic dysfunction in patients with newly diagnosed obstructive sleep apnoea and normal left ventricular ejection fraction. ScientificWorldJournal. 2014;2014:898746.

15. Glantz H, Thunstrom E, Johansson MC, Wallentin Guron C, Uzel H, Ejdeback J, et al. Obstructive sleep apnea is independently associated with worse diastolic function in coronary artery disease. Sleep Med. 2015;16(1):160-7.

16. Haruki N, Takeuchi M, Nakai H, Kanazawa Y, Tsubota N, Shintome R, et al.

17. Kawanishi Y, Ito T, Okuda N, Emura N, Hayashi T, Futai R, et al. Alteration of myocardial characteristics and function in patients with obstructive sleep apnea. Int J Cardiol. 2009;133(1):129-31.

18. Kim SH, Cho GY, Shin C, Lim HE, Kim YH, Song WH, et al. Impact of obstructive sleep apnea on left ventricular diastolic function. Am J Cardiol. 2008;101(11):1663-8.

19. Koga S, Ikeda S, Urata J, Kohno S. Effect of nasal continuous positive airway pressure in men on global left ventricular myocardial performance in patients with obstructive sleep apnea syndrome. Am J Cardiol. 2008;101(12):1796-800.

20. Lisi E, Faini A, Bilo G, Lonati LM, Revera M, Salerno S, et al. Diastolic dysfunction in controlled hypertensive patients with mild-moderate obstructive sleep apnea. Int J Cardiol. 2015;187:686-92.

21. Romero-Corral A, Somers VK, Pellikka PA, Olson EJ, Bailey KR, Korinek J, et al. Decreased right and left ventricular myocardial performance in obstructive sleep apnea. Chest. 2007;132(6):1863-70.

22. Wachter R, Luthje L, Klemmstein D, Luers C, Stahrenberg R, Edelmann F, et al. Impact of obstructive sleep apnoea on diastolic function. Eur Respir J. 2013;41(2):376-83.

23. Yang SQ, Han LL, Dong XL, Wang CY, Xia H, Liu P, et al. Mal-effects of obstructive sleep apnea on the heart. Sleep Breath. 2012;16(3):717-22.

24. Kane GC, Karon BL, Mahoney DW, Redfield MM, Roger VL, Burnett JC, Jr., et al. Progression of left ventricular diastolic dysfunction and risk of heart failure. JAMA. 2011;306(8):856-63. 
25. Redfield MM, Jacobsen SJ, Burnett JC, Jr., Mahoney DW, Bailey KR, Rodeheffer RJ. Burden of systolic and diastolic ventricular dysfunction in the community: appreciating the scope of the heart failure epidemic. JAMA. 2003;289(2):194-202.

\section{Hosted file}

subgroup analysis.pdf available at https://authorea.com/users/408502/articles/518488-sleepapnea-as-a-risk-factor-for-diastolic-dysfunction-a-systematic-review-and-meta-analysis

\section{Hosted file}

Figure 1.pdf available at https://authorea.com/users/408502/articles/518488-sleep-apnea-asa-risk-factor-for-diastolic-dysfunction-a-systematic-review-and-meta-analysis

\section{Hosted file}

Figure 2.pdf available at https://authorea.com/users/408502/articles/518488-sleep-apnea-asa-risk-factor-for-diastolic-dysfunction-a-systematic-review-and-meta-analysis

\section{Hosted file}

Figure 3.pdf available at https://authorea.com/users/408502/articles/518488-sleep-apnea-asa-risk-factor-for-diastolic-dysfunction-a-systematic-review-and-meta-analysis 